\title{
LEISHMANIOSE TEGUMENTAR AMERICANA NA REGIÃO NORDESTE DO ESTADO DE SÃO PAULO - BRASIL
}

\author{
M.G. Pignatti, R.C. Mayo, M.J.C.P. Alves, S.S.A.L Souza, \\ F. Macedo e R.M. Pereira
}

\begin{abstract}
Os autores descrevem um surto de leisbmaniose tegumentar americana ( LTA) instalado na região nordeste do Estado de São Paulo em 1992. Após a notificação de doze casos bumanos em uma área rural do município de Itupeva, foi realizado um inquérito epidemiológico, destacando-se 34\% de positividade da população ã Reação Intradérmica de Montenegro. Constatou-se uma grande diversidade da fauna flebotomínica local, com predominancia de L. migonei, L. intermedia e L. whitmani, tanto no domicilio, quanto na margem da mata. Ressalta-se a presença na margem da mata de L. longipalpis.
\end{abstract}

Palauras-chaves: Leishmaniose tegumentar. Epidemiologia. Flebótomos. Prevalência.

A leishmaniose tegumentar americana (LTA) tem sido referida no Estado de São Paulo desde meados do século, relacionada principalmente com o desflorestamento para a construção de rodovias e desenvolvimento agrícola ${ }^{14}$. Essas transformações, resultantes da atividade humana, influenciaram a incidência da doença, de modo a adquirir novos aspectos epidemiológicos, de acordo com as características das regiões em que a doença é assinalada atualmente ${ }^{6}$.

De doença classicamente profissional, atingindo adultos do sexo masculino, começa a incidir em todas as faixas etárias e em ambos os sexos. Atribui-se esta modificação, à adaptação das espécies transmissoras ao domicílio, permitindo a circulação de agentes etiológicos em ambientes extra florestais ${ }^{4619}$.

Segundo informações do Centro de Vigilância Epidemiológica (CVE), da Secretaria de Estado da Saúde de São Paulo, para onde são encaminhadas as notificações dos casos, a distribuição destes não é homogênea nas diversas regiões do Estado, havendo necessidade de elucidar aspectos locais da transmissão.

Nos últimos anos, tem sido constatada a expansão da LTA na região de Campinas,

\footnotetext{
Superintendência do Controle de Endemias, Vigilância Epidemiológica do Município de Itupeva e do ERSA-43 Jundiai, SP.

Endereço para correspondência: Dr ${ }^{a}$ Marta Gislene Pignatti. Rua São Carlos 546 - Vila Industrial, 13035-420 Campinas, SP, Tel: (0192) 31-7836.

Recebido para publicação em 22/11/94.
}

situada a nordeste do Estado de São Paulo, com registro de casos em vários municípios.

Em 1992, foram registrados doze casos de LTA na localidade rural - Bairro Quilombo- no município de Itupeva. Este episódio suscitou a realização de um inquérito epidemiológico, com o objetivo de conhecer o perfil da população residente, determinar a prevalência da infecção leishmaniótica e as espécies vetoras presentes na área.

\section{MATERIAL E MÉTODOS}

Área de estudo. O município de Itupeva está localizado a $23^{\circ} 3^{\prime}$ ao sul de latitude e a $47^{\circ}$ a oeste de longitude, distando $70 \mathrm{~km} \mathrm{da}$ cidade de São Paulo, com uma população de 18.065 habitantes $^{12}$.Segundo dados da Prefeitura Municipal de Itupeva, esse possui $166 \mathrm{~km}^{2}$ de zona rural e $30 \mathrm{~km}^{2}$ de zona urbana. Sua altitude é de $661 \mathrm{~m}$ acima do nível do mar. O clima predominante é o tipo mesotérmico de inverno seco. Constitui-se de um conjunto de pequenos morros arredondados de fundo úmido. Fragmentos de matas ciliares são encontrados ao longo do Rio Jundiaí, que atravessa todo o município. O solo atualmente é utilizado para o cultivo de uvas, plantio de cereais, flores, leguminosas e frutas. Encontram-se ainda na zona rural algumas casas de antigas colônias de fazendas de café e áreas com matas secundárias nas encostas dos morros. No passado foi explorada a extração de tungstênio e atualmente ocorre a extração de granito para calçamento . 
Pignatti MG, Mayo RC, Alves MJCP, Souza SSAL, Macedo F, Pereira RM. Leishmaniose tegumentar americana na região nordeste do Estado de São Paulo - Brasil. Revista da Sociedade Brasileira de Medicina Tropical 28:243-247, jul-set, 1995.

O Bairro Quilombo, de onde procedeu o maior número de casos (12) registrados no município, está situado a $10 \mathrm{~km}$ do centro de Itupeva. Está circundado por mata secundária e é cortado pelo Rio Jundiaí (Figura 1). Dispõe de várias residências de tijolos próximas uma das outras (Figura 2).

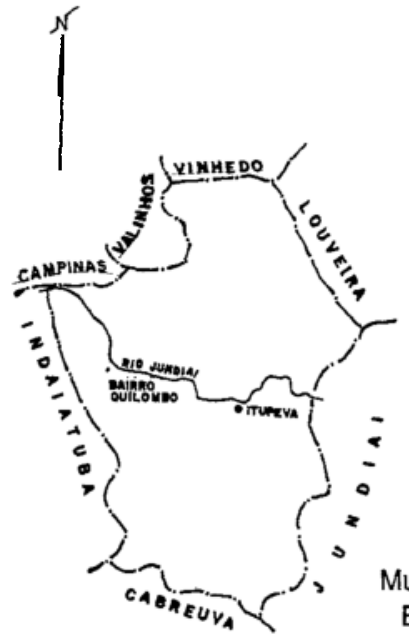

Município de Itupeva

Escala 1:250.000
Inquérito populacional. No período de agosto a setembro de 1992 , foram realizadas visitas casa a casa, registrando-se em um formulário informações sobre idade, sexo, profissão e tempo de moradia no local dos habitantes do bairro.

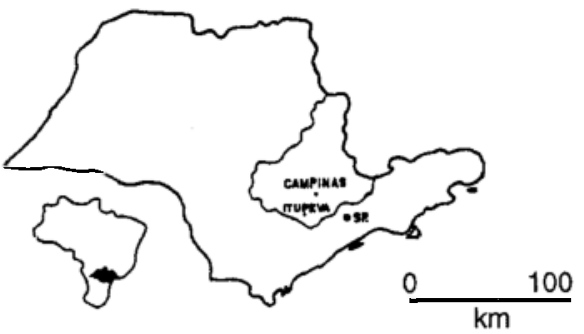

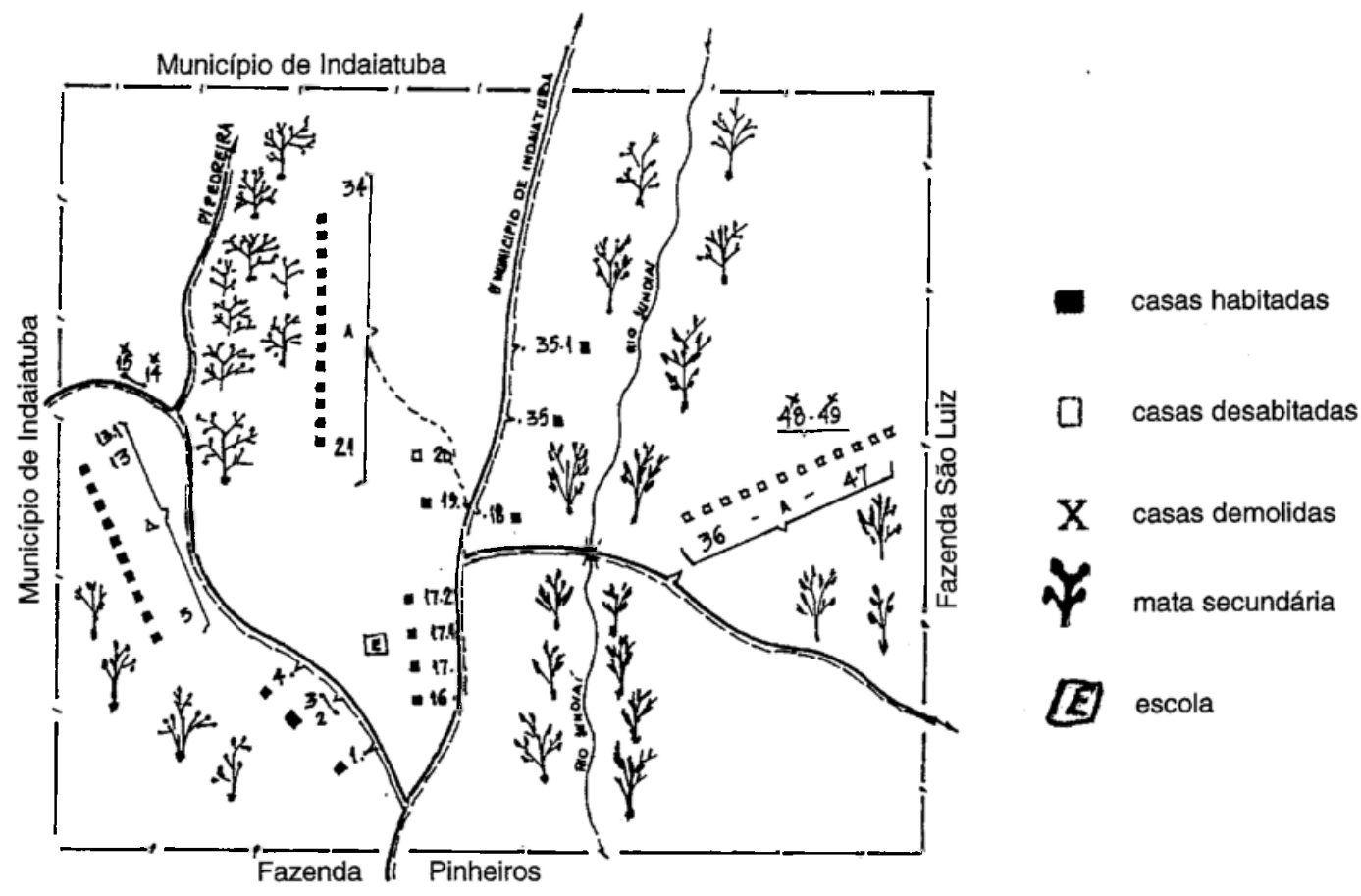

Figura 1: Localização geográfica da região nordeste do Estado, do município de Itupeva e do Bairro Quilombo. 
Pignatti MG, Mayo RC, Altes MJCP, Souza SSAL, Macedo F, Pereira RM. Leishmaniose tegumentar americana na região nordeste do Estado de São Paulo - Brasil. Retista da Sociedade Brasileira de Medicina Tropical 28:243-247, jul-set, 1995.

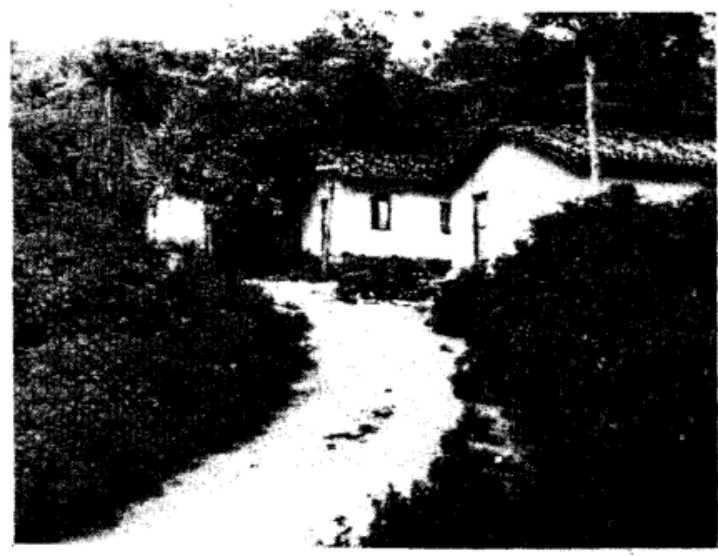

Figura 2: Vista parcial do Bairro Quilombo, com detalbes da conformação das residências.

Teste de Prevalência. Foi realizada a intradermorreação de Montenegro nos habitantes do Bairro. $O$ antígeno de Leishmania foi fornecido pelo Instituto Adolfo Lutz, contendo $40 \mathrm{~g} \mathrm{~N} / \mathrm{ml}^{13}$ e aplicado na face anterior do antebraço direito, na dose de 0,1 $\mathrm{ml}$. A leitura das reaçōes foram feitas após 72 horas, identificando-se as endurações com dedo indicador, marcando com uma caneta e medindo o diâmetro no sentido transverso, com régua utilizada para teste de $P P D$ (Derivado Proteico Purificado), utilizado para BCGID , conforme Manual do Centro de Vigilância Epidemiológica da Secretaria de Estado da Saúde de São Paulo. Foram consideradas positivas as endurações com diâmetro iguais ou superiores a $5 \mathrm{~mm}^{13}$.

Inquêrito entomológico. As coletas de flebotomíneos foram realizadas nas paredes internas das residências, com auxílio de tubo contendo vapores de clorofórmio ${ }^{3}$. Armadilhas luminosas tipo $\mathrm{CDC}^{18}$ foram instaladas no intradomicílio, abrigo de animais situados no peridomicílio e nas margens da mata. Realizou-se, ainda, coletas com armadilhas de Shannon ${ }^{317}$, instaladas a $50 \mathrm{~m}$ das residências. As coletas foram realizadas ao mesmo tempo, no período das 18 às 21 horas.

\section{RESULTADOS}

O recenseamento realizado no Bairro Quilombo abrangeu 34 habitações, num total de 144 individuos (Tabela 1). As atividades ocupacionais dos indivíduos estavam assim distribuídas: $29,2 \%$ realizavam atividades nas pedreiras, extraindo pedras para calçamento;
$39 \%$ eram estudantes; $22 \%$ do lar; $4,9 \%$ de aposentados e o restante ( $4,9 \%)$ realizavam atividades diversas (comerciante, diarista, merendeira, caseiro).

As habitações estavam alugadas aos moradores e todas elas eram de tijolos rebocados. sem forração. Apenas 50\% destas possuíam sanitário. Verificou-se a presença de 15 galinheiros próximos as habitaçôes e a existência de 40 cães domésticos.

Dos 144 indivíduos entrevistados, 100 destes submeteram-se ao teste intradérmico de Montenegro. Os demais moradores estavam ausentes nos dias definidos para a realização do inquérito. Com relação ao resultado do teste, observou-se que das 100 pessoas examinadas, 34\% apresentaram resultado positivo. A distribuição quanto ao sexo mostrou uma igualdade percentual (50\%) entre os mesmos. $\mathrm{Na}$ faixa etária, nota-se a predominância de resultado positivo a partir dos 21 anos, registrando-se nas faixas subsequentes um percentual em torno de $50 \%$ (Tabela 1). Dos individuos positivos, $52 \%$ referem moradia no mesmo local há pelo menos 11 anos, $32 \%$ de 1 a 5 anos, $12 \%$ de 6 a 10 anos e $3 \%$ há menos de 1 ano.

A pesquisa entomológica apontou a coleta de 306 flebotomíneos de 12 espécies diferentes. Destaca-se a presença de 3 espécies predominantes no domicílio: L. migonei (38\%), L. intermedia (25\%) e L. whitmani (15\%). Em abrigos de animais foi registrada a presença de L. migonei (45\%), L. whitmani (23\%) e $L$. firmatoi $(14 \%)$. Nas margens da mata foram encontradas espécies de L. intermedia (45\%) e $L$. migonei (18\%) . Ressalta-se a presença de $L$. longipalpis ( $1 \%$ ) coletada na margem da mata (Tabela 2). Não foi realizada dissecção dos flebotomíneos para pesquisar se estavam infectados por falta de apoio laboratorial.

\section{DISCUSSÃO}

Através desses dados, pondera-se que a doença está assumindo aspectos compatíveis com outras áreas do Estado ${ }^{4619}$, visto que é decorrente da adaptação das espécies vetoras em áreas que sofreram intervenção humana. Todavia, a detecção de algumas principais espécies vetoras da LTA na composição flebotominica local merece novos estudos, face a necessidade de aprofundar os dados para esclarecer o padrão de transmissão em 
Pignatti MG, Mayo RC, Alues MJCP, Souza SSAL, Macedo F, Pereira RM. Leishmaniose tegumentar americana na região nordeste do Estado de São Paulo - Brasil. Revista da Sociedade Brasileira de Medicina Tropical 28:243-247, jul-set, 1995.

Itupeva. Mesmo assim, a presença de $L$. intermedia $\mathrm{e} L$. whitmani no interior da habitação (Tabela 2) e a taxa de prevalência em menores de 10 anos corrobora com a suspeita de que parte da transmissão tenha ocorrido no intradomicílio 35791114 . Por outro lado, em que pese homens e mulheres com a mesma taxa de infecção, o envolvimento desses indivíduos com a mata, desenvolvendo atividades de exploração de pedras, os coloca expostos ao risco de adquirir a doença em ambos os ambientes (domiciliar e extra domiciliar).

Com referência às espécies (Tabela 2), observa-se aquelas com papel epidemiológico mais definido, tais como a $L$. migonei, $L$. intermedia, L. whitmani encontradas em maior densidade e descritas como vetoras da L.(V) braziliensis no Estado de São Paulo ${ }^{15}$.

Embora Gomes ${ }^{8}$ já tenha assinalado uma similaridade de comportamento de L. migonei e $L$. fischeri no domicílio humano com a $L$. intermedia, a incriminação dessas espécies na transmissão local, deverá ser melhor investigada.

Levando-se em conta que a intradermorreação de Montenegro indica que o indivíduo já foi sensibilizado e que às vezes as pessoas portadoras das doenças não reagem ao teste ${ }^{2}$, especialmente na fase precoce, encontrou-se um resultado de $34 \%$ de positividade semelhante ao encontrado por Tolezano ${ }^{20}$ no município de São Roque, Estado de São Paulo, com uma positividade de 33,6\% da população da área considerada de risco e um pouco acima do encontrado por Gomes ${ }^{10}$ no Vale do Ribeira (25,5\%).

Cabe ressaltar que, as manifestações clínicas dos casos caracterizaram-se por lesões ulceradas dolorosas, com contornos circulares, bordas altas e infiltradas, fundo com granulações grandes $e$ vermelho viva $e$ recobertas por exudato, às vezes seroso, às vezes sero-purulento. Em $75 \%$ dos casos as lesões eram múltiplas, localizadas preferencialmente nos braços e nas pernas. Os casos foram submetidos à reação de Montenegro, sendo todos positivos (enduração superior a $5 \mathrm{~mm}$ ). Colheu-se material para cultura e em três deles, esta resultou positiva para Leishmania. Apesar da identificação da espécie ter sido infrutífera, supõe-se tratar de $L$. $(V)$ braziliensis, particularmente quanto as manifestações clínicas, que se assemelham a casos onde há ocorrência exclusiva desse agente $^{2}$.

Finalmente, os resultados apresentados no presente estudo, acrescentam subsídios ao conhecimento da expansão epidemiológica da LTA no Estado de São Paulo, além de ensejar novas investigações sobre o comportamento $\mathrm{e}$ competência vetorial de flebotomíneos, bem como tentar identificar reservatórios nesta área. Simultaneamente, busca-se subsídios que possam aprimorar estratégias de controle da transmissão.

\section{SUMMARY}

An outbreak of American Cutaneous Letshmaniasis (ACL) occurring in the Nortbeast region of São Paulo state in 1992 is described. After the notification of the 12 buman cases, a skin test survey of the local population was carried out in a rural area of the Itupeva municipality. The survey consisted of 144 interviews and 100 clinical examinations using the Montenegro skin test (MST). A prevalence of $34 \%$ positive MST was encountered. The predominant species of sandflies captured both in domestic and nearby areas of secondary vegetation were $\mathrm{L}$. intermedia, $\mathrm{L}$. whitmani and $\mathrm{L}$. migonei. The presence of $\mathrm{L}$. longipalpis in the nearby area of secondary vegetation was also registered.

Key-words: Cutaneous leishmaniasis. Epidemiology. Sandflies. Prevalence.

\section{AGRADECIMENTOS}

À Prof $^{\mathbf{a}} \operatorname{Dr}^{\mathbf{a}}$ Eunice A. B. Galati pela identificação dos flebotomíneos, ao Prof. Dr. Almério de Castro Gomes e Dra. Dalva M. Wanderley pela revisão do trabalho e ao Rafael P. Frederice pelo auxílio na digitação.

\section{REFERÊNCIAS BIBLIOGRÁFICAS}

1. Dourado MIC, Noronha CV, Alcântara N, Ichihara MYT, Loureiro S. Epidemiologia da leishmaniose tegumentar e suas relações com a lavoura e o garimpo, em localidade do Estado da Bahia (Brasil). Revista de Saúde Pública 23:2-8, 1989.

2. Falqueto A, Sessa PA. Leishmaniose tegumentar americana. In: Veronesi $\mathrm{R}$ (ed) Doenças Infecciosas e Parasitárias. Editora Guanabara Koogan, $8^{a}$ edição, Rio de Janeiro p. 750-761, 1991

3. Forattini OP. Entomologia Médica. Editora Universidade de São Paulo, São Paulo p.598-607, 1962. 
Pignatti MG, Mayo RC, Alues MJCP, Souza SSAL, Macedo F, Pereira RM. Leishmaniose tegumentar americana na região nordeste do Estado de São Paulo - Brasil. Revista da Sociedade Brasileira de Medicina Tropical 28:243-247, jul-set, 1995.

4. Forattini OP, Rabello EX, Serra OP, Cotrim MD, Galati EAB, Barata JMS. Observações sobre a transmissão da leishmaniose tegumentar americana no Estado de São Paulo, Brasil. Revista de Saúde Pública 10:30-43, 1976

5. Forattini OP, Santos MR. Nota sobre infecção natural de Pblebotomus intermedius, Luta e Neiva, 1912, por formas leptomonas em foco de leishmaniose tegumentar americana. Arquivos de Higiene e Saúde Pública 17:171-174, 1952

6. Gomes AC. Perfil epidemiológico da leishmaniose tegumentar americana no Brasil. Anais Brasileiros de Dermatologia 67:55-60, 1992

7. Gomes AC, Galati EAC. Aspectos ecológicos da leishmaniose tegumentar americana. 7 Capacidade vetorial flebotomínea em ambiente florestal primário do sistema da Serra do Mar , Região do Vale do Ribeira, Estado de São Paulo, Brasil. Revista de Saúde Pública 23:136-142, 1989.

8. Gomes AC, Rabello EX, Santos JLF, Galati EAB. Aspectos ecológicos da leishmaniose tegumentar americana. 1 Estudo experimental da freqüência de flebotomíneos a ecótopos artificiais com referência especial a Psycbodopygus intermedius. Revista de Saúde Pública 14:540. 556,1980

9. Gomes AC, Rabello EX, Santos JLF, Galati EAB. Aspectos ecológicos da leishmaniose tegumentar americana. 3 Observações sobre o ritmo diário da atividade de Psicbodopygus intermedius em ambiente florestal e extra florestal. Revista de Saúde Pública 17:23-30, 1983

10. Gomes AC, Yamamoto YI, Capinzaiki AN, Amaral NMM, Guimarães AJG. Aspectos epidemiológicos da leishmaniose tegumentar americana. Prevalência /incidência da infecção humana nos municípios de Pedro de Toledo e Miracatu, São Paulo, Brasil. Revista do Instituto de Medicina Tropical de São Paulo 34:149-158, 1992

11. Hoch A, Ryan L, vexenat JA, Rosa ACOC, Barreto AC Isolation of Leishmania braziliensis braziliensis and other trypanosomatids from
Phlebotomine in a mucocutaneous leishmaniasis endemic area, Bahia, Brazil. Memótias do Instituto Oswaldo Cruz Rio de Janeiro 81(supl):62,1986.

12. Instituto Brasileiro de Geografia e Estatística (IBGE). Censo Demográfico, 1991.

13. Melo MN, Mayrink W, Costa CA, Magalhães PA, Dias M, Williams P, Araújo FG, Coelho MV, Batista SM. Padronização do antígeno de Montenegro. Revista do Instituto de Medicina Tropical de São Paulo 19:161-164, 1977.

14. Pessoa SB, Barreto MP. Leishmaniose tegumentar americana. Rio de Janeiro, Ministério da Educação, 1948.

15. Pessoa SB, Pestana BR. Infecção natural do Pblebotomus migonei por formas de leptomonas, provavelmente da Leishmania braziliensis. Acta médica 5:106-111, 1940.

16. Ryan L, Vexenat A, Marsden PD, Laison R, Shaw JJ. The importance of rapid diagnosis of new cases of cutaneous leismaniasis in pin-pointing the sandfly vector. Transactions of the Royal Society of Tropical Medicine and Hygiene 84:786, 1990.

17. Sherlock JA, Pessoa SB. Métodos Práticos para a captura de flebótomos. Revista Brasileira de Biologia 24:331-340, 1964.

18. Sudia WD, Chamberlain RN. Battery operated light trap and improved model. Mosquito News 22:126-129, 1962.

19. Tolezano JE, Macoris SAG, Diniz JMP. Modificação da epidemiologia da leishmaniose tegumentar americana no Vale do Ribeira, Estado de São Paulo, Brasil. Revista do Instituto Adolfo Lutz 40:49-54, 1980.

20. Tolezano JE, Reina RB, Taniguchi HH, Araújo MFL, Pinheiro M, D Andrade OM, Balanço JMF, Wanderley DMV, Correa FMA. American Cutaneous Leishmaniasis in São Paulo StateEpidemiologic studies in São Roque country, 1987-1989. Procedings of 16 th annual meeting on basic research in Chagas disease Caxambú, Minas Gerais,Brasil p. 34, 1989. 